\title{
An algebraic formulation of static isotropy and design of statically isotropic 6-6 Stewart platform manipulators
}

\author{
Sandipan Bandyopadhyay* \\ Ashitava Ghosal \\ Department of Engineering Design \\ Department of Mechanical Engineering \\ Indian Institute Technology- Madras \\ Indian Institute of Science \\ Chennai 600036, India \\ Bangalore 560012, India
}

\begin{abstract}
In this paper, we present an algebraic method to study and design spatial parallel manipulators that demonstrate isotropy in the force and moment distributions. We use the force and moment transformation matrices separately, and derive conditions for their isotropy individually as well as in combination. The isotropy conditions are derived in closed-form in terms of the invariants of the quadratic forms associated with these matrices. The formulation is applied to a class of Stewart platform manipulator, and a multi-parameter family of isotropic manipulator is identified analytically. We show that it is impossible to obtain a spatially isotropic configuration within this family. We also compute the isotropic configurations of an existing manipulator and demonstrate a procedure for designing the manipulator for isotropy at a given configuration.
\end{abstract}

\footnotetext{
*Corresponding author, email: sandipan@iitm.ac.in.
} 


\section{Introduction}

Isotropy is one of the common measures of performance of a manipulator. In the case of sixdegrees-of-freedom (DOF) spatial manipulators, the term isotropy is generally used in the context of kinematics. However, in practice, the concept of twist-wrench duality is used to analyse the $6 \times 6$ wrench transformation matrix $\boldsymbol{H}$, to obtain conditions such that this matrix has identical singular values (see, e.g., [1]). A consequence of this approach is the concurrence of kinematic and static isotropy, where the later implies the ability of the manipulator endeffector to resist forces and moments equally well in all spatial directions. Among the spatial parallel manipulators, the Stewart platform manipulator (SPM) has been studied by several researchers for isotropy $[1,2,3,4]$. However, to the best of our knowledge, no mechanically feasible, non-singular isotropic configuration has been obtained for a manipulator of this class. Further, it may be noted that the $3 \times 6$ sub-matrices of $\boldsymbol{H}$ pertaining to the force and moment parts have different physical dimensions for an SPM, therefore the physical significance of the singular values of $\boldsymbol{H}$ is not clear.

In this paper, we present a formulation for the study of static isotropy. Our approach is to analyse the above-mentioned force and moment transformation matrices separately. We form the conditions for the force and moment isotropy in terms of algebraic equations involving the elements of the respective transformation matrices. We solve these equations in closed form to obtain a multi-parameter family of kinematically valid configurations showing combined force and moment isotropy. We also present examples of isotropic configurations for an existing manipulator, as well as demonstrate the design for isotropy at a given configuration within the family mentioned above.

The paper is organised as follows: in section 2, we present the general formulation of static isotropy of a spatial manipulator, followed by its application to the semi-regular Stewart platform manipulator (SRSPM) in section 3. In section 4, we identify, in closed form, a family of SRSPM showing combined spatial isotropy. This is followed by a numerical example, where we find such configurations for an SRSPM. In section 5, we study various notions of isotropy and discuss their inter-relations. We also prove that it is impossible to attain a spatially 
isotropic manipulator with the family of configurations obtained in section 4 . In section 5 we present the method of design for isotropy at a given configuration, and illustrate it with an example. Finally, in section 6 , we present the conclusions.

\section{Formulation}

In this section, we derive the isotropy conditions of a general manipulator from its wrench transformation matrix. First we describe the formulation for obtaining the distributions of the force and moment resultants on the moving platform. We follow the approach presented in [5] in the context of the linear and angular velocity distributions of the moving platform. Using this approach, the said distributions are obtained from the solution of eigenproblems of two symmetric matrices. The conditions for force and moment isotropy are then derived in terms of algebraic equations involving the coefficients of the characteristic polynomials associated with the above eigenproblems. We assume in the following that the resultant force on the top platform, $\boldsymbol{F}$, and the corresponding moment (referred to the centre of the moving platform), $\boldsymbol{M}$, are available via linear maps of the actuator efforts (e.g., leg forces in the case of a platform-type parallel manipulator), $\boldsymbol{f}_{i} \cdot{ }^{1}$ Therefore we can write $\boldsymbol{F}$ and $\boldsymbol{M}$ in terms of the respective equivalent transformation matrices:

$$
\begin{gathered}
\boldsymbol{F}=\boldsymbol{H}_{\boldsymbol{F}} \boldsymbol{f} \\
M=\boldsymbol{H}_{M} \boldsymbol{f}
\end{gathered}
$$

We analyse the properties of the above two linear maps using well-known tools of linear algebra (see, e.g., [6]). Following the general formulation given in Appendix A, we get two

\footnotetext{
${ }^{1}$ Obtaining such a map is trivial for purely parallel manipulators. However, for hybrid manipulators, there can be significant difficulty in taking the reactions at the passive joints into account while computing the effect of the actuator effort on the end-effector.
} 
eigenproblems respectively:

$$
\begin{gathered}
\boldsymbol{g}_{\boldsymbol{F}} \boldsymbol{f}=\lambda \boldsymbol{f} \\
\boldsymbol{g}_{\boldsymbol{M}} \boldsymbol{f}=\lambda_{M} \boldsymbol{f}
\end{gathered}
$$

where $\boldsymbol{g}_{\boldsymbol{F}}=\boldsymbol{H}_{\boldsymbol{F}}^{T} \boldsymbol{H}_{\boldsymbol{F}}$ and $\boldsymbol{g}_{\boldsymbol{M}}=\boldsymbol{H}_{\boldsymbol{M}}^{T} \boldsymbol{H}_{\boldsymbol{M}}$. These eigenproblems have the following characteristics:

- The eigenvalues $\lambda, \lambda_{M}$ are real and nonnegative.

- At the most three of the eigenvalues are nonzero in each case, as the rank of $\boldsymbol{H}_{\boldsymbol{F}}$ or $\boldsymbol{H}_{\boldsymbol{M}}$ can not exceed three. Therefore, if $\operatorname{dim}\left(\boldsymbol{g}_{\boldsymbol{F}}\right)=n$ with $n>3$, at least $(n-3)$ eigenvalues of $\boldsymbol{g}_{\boldsymbol{F}}$ are zeros- and the same applies to $\boldsymbol{g}_{\boldsymbol{M}}$ as well.

The characteristic equation of $\boldsymbol{g}_{\boldsymbol{F}}$ may be written with real coefficients $a_{i}$ as:

$$
0= \begin{cases}\lambda^{2}+a_{1} \lambda+a_{2}, & n=2 \\ \lambda^{3}+a_{1} \lambda^{2}+a_{2} \lambda+a_{3}, & n=3 \\ \lambda^{n-3}\left(\lambda^{3}+a_{1} \lambda^{2}+a_{2} \lambda+a_{3}\right), & n>3\end{cases}
$$

The characteristic equation of $\boldsymbol{g}_{\boldsymbol{M}}$ has exactly the same form as above. However, we use the notations $b_{i}$ for the coefficients, and $\lambda_{M}$ for the eigenvalues in that case. From linear algebra, isotropy of $\boldsymbol{H}_{\boldsymbol{F}}$ and $\boldsymbol{H}_{\boldsymbol{M}}$ imply, respectively:

$$
\begin{aligned}
& \lambda_{i}=\left\|\boldsymbol{F}^{*}\right\|^{2} \\
& \lambda_{\boldsymbol{M} i}=\left\|\boldsymbol{M}^{*}\right\|^{2} \quad i=1, \ldots, n
\end{aligned}
$$

where $\left(.^{*}\right)$ indicates the extreme value of a quantity. Under this condition, the force ellipsoid, (the ellipsoid corresponding to $\boldsymbol{F}$ ) reduces to a sphere of radius $\left\|\boldsymbol{F}^{*}\right\|$. Similarly, the moment ellipsoid reduces to a sphere of radius $\left\|M^{*}\right\|$. This implies that the nontrivial roots of equation (4) should be equal, and not all of $a_{1}, a_{2}, a_{3}$ can be zero. ${ }^{2}$ The nontrivial roots

\footnotetext{
${ }^{2}$ It may be noted here that the coefficients $a_{i}, b_{i}$ can be computed in closed form as described in Appendix B.
} 
of equation (4) can also be obtained explicitly in terms of $a_{i}$ using Sridhar Acharya's and Cardan's formulæ for the quadratic and cubic cases respectively (see, e.g., [7]). However, it is not required to compute the roots explicitly in order to obtain the conditions for isotropy from their equality. Instead, those conditions can be easily formed as algebraic equations in the coefficients $a_{i}$ etc. as follows. For the case $n=2$, we equate the discriminant to zero and obtain the following condition:

$$
a_{1}^{2}-4 a_{2}=0
$$

For the case $n \geq 3$, we consider the nontrivial cubic part of equation (4):

$$
\lambda^{3}+a_{1} \lambda^{2}+a_{2} \lambda+a_{3}=0
$$

Using the standard transformation $\lambda=z-\frac{a_{1}}{3}$, the quadratic term may be eliminated to obtain the cubic in the form: $z^{3}+P z+Q=0$ (see, e.g., [7]). It is obvious that if $P=Q=0$, then $z=0$, and hence equation (7) has the repeated roots $\lambda_{i}=-\frac{a_{1}}{3}, i=1,2,3$. In terms of the coefficients of the original cubic equation (7), the conditions for equal roots are:

$$
\begin{aligned}
& 3 a_{2}-a_{1}^{2}=0 \\
& 2 a_{1}^{3}-9 a_{1} a_{2}+27 a_{3}=0
\end{aligned}
$$

Further, if we solve the above equations exactly in the symbolic form, then the second of them can be simplified using the first, yielding the pair of equations below:

$$
\begin{aligned}
& 3 a_{2}-a_{1}^{2}=0 \\
& 27 a_{3}-a_{1}^{3}=0
\end{aligned}
$$

The conditions for moment isotropy can be obtained in the same fashion. In the following, we list down the conditions for the different types of isotropy considered in this paper.

A. Force $\left(\boldsymbol{F}\right.$-isotropy): $\boldsymbol{H}_{\boldsymbol{F}}$ is isotropic.

$$
\left.\begin{array}{l}
a_{1}^{2}-4 a_{2}=0, \\
3 a_{2}-a_{1}^{2}=0 \\
27 a_{3}-a_{1}^{3}=0
\end{array}\right\} \quad n \geq 3
$$


B. Moment ( $\boldsymbol{M}$-isotropy): $\boldsymbol{H}_{\boldsymbol{M}}$ is isotropic.

$$
\left.\begin{array}{l}
b_{1}^{2}-4 b_{2}=0, \\
3 b_{2}-b_{1}^{2}=0 \\
27 b_{3}-b_{1}^{3}=0
\end{array}\right\} n \geq 3
$$

C. Combined: Both $\boldsymbol{H}_{\boldsymbol{F}}, \boldsymbol{H}_{\boldsymbol{M}}$ are isotropic. The conditions that apply in this case are simply the union of the conditions in cases A and B.

$$
\left.\begin{array}{l}
a_{1}^{2}-4 a_{2}=0 \\
b_{1}^{2}-4 b_{2}=0
\end{array}\right\}
$$

\section{Isotropy conditions of an SRSPM}

In this section, we apply the theory developed in section 2 to formulate the isotropy conditions of an SRSPM. In addition to its wide-spread technical applications, the other motivations to choose this manipulator as our example are: (a) it is probably the most well-studied spatial parallel manipulator (see section 1 for some of the references) (b) no feasible configuration of any Stewart platform manipulator demonstrating combined static isotropy is reported in literature to the best of our knowledge. The manipulator along with the frames of reference used is shown in figure 1. The bottom platform, shown in figure 2, has the legs arranged in a circle, with each pair lying symmetrically on either side of three axes of symmetry in the plane. The axes are $\frac{2 \pi}{3}$ apart from each other, while the adjacent pair of legs have an angular spacing $2 \gamma_{b}$. Without any loss of generality, we scale the radius of the circumcircle of the bottom platform, $r_{b}$, to unity, thus eliminating one parameter from all subsequent analysis, and rendering all other length parameters used in this paper dimensionless ${ }^{3}$. The

\footnotetext{
${ }^{3}$ We use radians for the angular unit in this paper, while the unit for the base radius can be chosen as convenient.
} 


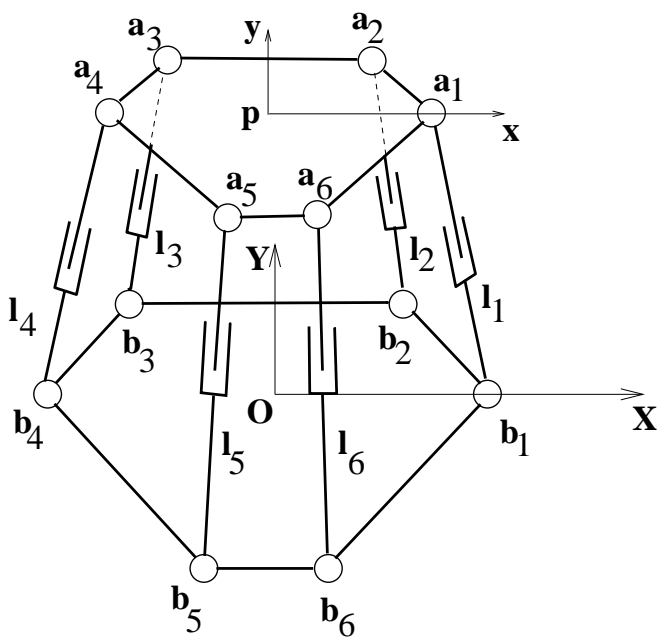

(a) The manipulator

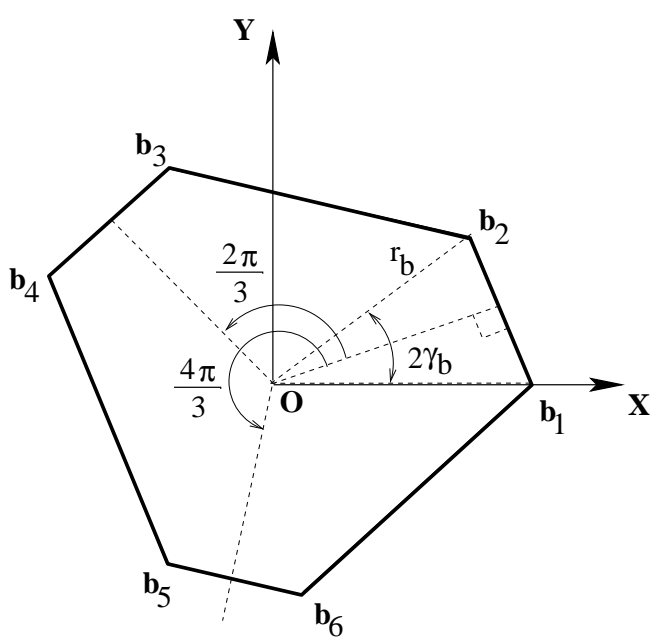

(b) Bottom platform

Figure 1: Geometry of the semi-regular Stewart platform manipulator

top platform geometry is similar, except that the radius of its circumcircle is $r_{t}$, and the leg spacing is $2 \gamma_{t}$.

The kinematic constraints defining the manipulator are written in the task-space variables. The centre of the top platform is described in the base frame as $\boldsymbol{p}=(x, y, z)^{T}$. The top platform orientation is described by the matrix $\boldsymbol{R} \in S O(3)$, where $\boldsymbol{R}=\boldsymbol{R}_{z}(\phi) \boldsymbol{R}_{x}\left(\theta_{x}\right) \boldsymbol{R}_{y}\left(\theta_{y}\right)^{4}$. The loop-closure equations are written as:

$$
\boldsymbol{p}+\boldsymbol{R} \boldsymbol{a}_{i}-\boldsymbol{b}_{i}-l_{i} \boldsymbol{s}_{i}=\mathbf{0}, \quad i=1, \ldots, 6
$$

where $l_{i}$ denotes the length of the $i$ th leg and $\boldsymbol{a}_{i}, \boldsymbol{b}_{i}$ locate the leg connection points with respect to the platform centres in the respective frames (see figure 1), and $\boldsymbol{s}_{i}$ denotes the $i$ th screw axis along the respective leg. The screw axis can be written in terms of the task-space variables and actuated variables as:

$$
\boldsymbol{s}_{i}=\frac{1}{l_{i}}\left(\boldsymbol{p}+\boldsymbol{R} \boldsymbol{a}_{i}-\boldsymbol{b}_{i}\right), i=1, \ldots, 6
$$

\footnotetext{
${ }^{4}$ In this paper, we denote the rotation about the axis $\boldsymbol{X}$ through an angle $\theta$ as $\boldsymbol{R}_{x}(\theta)$ etc.
} 
The actuation force along the $i$ th leg can be written as $\boldsymbol{F}_{i}=f_{i} \boldsymbol{s}_{i}$, where $f_{i}$ denotes the sense and magnitude of the force. In terms of the force transformation matrix, the resultant force on the top platform can be written as:

$$
\boldsymbol{F}=\boldsymbol{H}_{\boldsymbol{F}} \boldsymbol{f}
$$

where, $\boldsymbol{f}=\left(f_{1}, f_{2}, f_{3}, f_{4}, f_{5}, f_{6}\right)^{T}$ is the vector of leg forces, and the matrix $\boldsymbol{H}_{\boldsymbol{F}}$ is given by:

$$
\boldsymbol{H}_{\boldsymbol{F}}=\left(\begin{array}{ccc}
\frac{1}{l_{1}}\left(\boldsymbol{p}+\boldsymbol{R} \boldsymbol{a}_{1}-\boldsymbol{b}_{1}\right)_{1} & \ldots & \frac{1}{l_{6}}\left(\boldsymbol{p}+\boldsymbol{R} \boldsymbol{a}_{6}-\boldsymbol{b}_{6}\right)_{1} \\
\frac{1}{l_{1}}\left(\boldsymbol{p}+\boldsymbol{R} \boldsymbol{a}_{1}-\boldsymbol{b}_{1}\right)_{2} & \ldots & \frac{1}{l_{6}}\left(\boldsymbol{p}+\boldsymbol{R} \boldsymbol{a}_{6}-\boldsymbol{b}_{6}\right)_{2} \\
\frac{1}{l_{1}}\left(\boldsymbol{p}+\boldsymbol{R} \boldsymbol{a}_{1}-\boldsymbol{b}_{1}\right)_{3} & \ldots & \frac{1}{l_{6}}\left(\boldsymbol{p}+\boldsymbol{R} \boldsymbol{a}_{6}-\boldsymbol{b}_{6}\right)_{3}
\end{array}\right)
$$

where $(\cdot)_{i}$ denotes the $i$ th component of the vector ' $\because$ '. Moment imparted on the top platform due to the force along the $i$ th leg can be written as $\boldsymbol{M}_{i}=\left(\boldsymbol{R} \boldsymbol{a}_{i}\right) \times f_{i} \boldsymbol{s}_{i}$. Using the expression for $\boldsymbol{s}_{i}$ from equation (17), $\boldsymbol{M}_{i}$ may be written as $\boldsymbol{M}_{i}=\frac{f_{i}}{l_{i}}\left(\boldsymbol{R} \boldsymbol{a}_{i}\right) \times\left(\boldsymbol{p}-\boldsymbol{b}_{i}\right)$. Therefore the resultant moment $\boldsymbol{M}$ can be written in terms of the moment transformation matrix $\boldsymbol{H}_{\boldsymbol{M}}$ as

$$
\begin{aligned}
& \boldsymbol{M}=\boldsymbol{H}_{\boldsymbol{M}} \boldsymbol{f} \\
& \boldsymbol{H}_{\boldsymbol{M}}=\left(\begin{array}{lll}
\frac{1}{l_{1}}\left(\boldsymbol{R} \boldsymbol{a}_{1} \times\left(\boldsymbol{p}-\boldsymbol{b}_{1}\right)\right)_{1} & \ldots & \frac{1}{l_{6}}\left(\boldsymbol{R} \boldsymbol{a}_{6} \times\left(\boldsymbol{p}-\boldsymbol{b}_{6}\right)\right)_{1} \\
\frac{1}{l_{1}}\left(\boldsymbol{R} \boldsymbol{a}_{1} \times\left(\boldsymbol{p}-\boldsymbol{b}_{1}\right)\right)_{2} & \ldots & \frac{1}{l_{6}}\left(\boldsymbol{R} \boldsymbol{a}_{6} \times\left(\boldsymbol{p}-\boldsymbol{b}_{6}\right)_{2}\right. \\
\frac{1}{l_{1}}\left(\boldsymbol{R} \boldsymbol{a}_{1} \times\left(\boldsymbol{p}-\boldsymbol{b}_{1}\right)\right)_{3} & \ldots & \frac{1}{l_{6}}\left(\boldsymbol{R} \boldsymbol{a}_{6} \times\left(\boldsymbol{p}-\boldsymbol{b}_{6}\right)_{3}\right.
\end{array}\right)
\end{aligned}
$$

It may be noted that the use of equation (17) ensures that the expressions of $\boldsymbol{H}_{\boldsymbol{F}}$ and $\boldsymbol{H}_{\boldsymbol{M}}$ are kinematically consistent, i.e., the loop closure equations are automatically satisfied when they are cast in this form.

The conditions for static isotropy are obtained from $\boldsymbol{H}_{\boldsymbol{F}}, \boldsymbol{H}_{\boldsymbol{M}}$ following the previous section. The computational steps involved for all the cases A, B, and C are summarised below.

1. Form the symmetric matrix $\boldsymbol{g}_{\boldsymbol{F}}=\boldsymbol{H}_{\boldsymbol{F}}^{T} \boldsymbol{H}_{\boldsymbol{F}}$

2. Form the symmetric matrix $\boldsymbol{g}_{\boldsymbol{M}}=\boldsymbol{H}_{\boldsymbol{M}}^{T} \boldsymbol{H}_{\boldsymbol{M}}$ 
3. Compute the coefficients of the characteristic equations of $\boldsymbol{g}_{\boldsymbol{F}}, \boldsymbol{g}_{\boldsymbol{M}}$ using equation (45) (see Appendix B).

4. Use equations (15) or any subset of the same, as appropriate for the different cases of static isotropy.

\section{Analytical results on the isotropy of an SRSPM}

We now describe some analytical results for the different cases of isotropy of the SRSPM using the formulation developed in the last section. The independent variables involved in the isotropy equations are the position of the top platform $\boldsymbol{p}=(x, y, z)^{T}$, the orientation variables $\alpha, \beta, \phi$, and the architecture variables $r_{t}, \gamma_{b}$ and $\gamma_{t}$.

\subsection{Architecture, configuration constraints}

The natural restrictions on the architecture parameters for a mechanically feasible design would be the following:

- $\overline{r_{t}} \geq r_{t} \geq \underline{r_{t}}$ where $\overline{r_{t}}, \underline{r_{t}}>0$ are two prescribed limits. We adopt in this work $\overline{r_{t}}=1, \underline{r_{t}}=1 / 4$.

- $\pi / 3 \geq \gamma_{b}, \gamma_{t} \geq 0$. At both the ends, the hexagonal platforms reduce to triangles, and beyond these limits the leg connection points with the platforms cross over, and the legs can interfere mutually.

- The moving platform is above the fixed one, i.e., $z>0$.

- $\gamma_{b} \neq \gamma_{t}$. If the platforms are scaled versions of each other, the manipulator is architecturally singular $[8,9]$.

Any solution for the architecture within these restrictions would be termed as feasible or valid. Other mechanical constraints, such as joint limits, leg limits, and physical dimensions of the legs etc. are not considered in the present work. As a result, we do not impose 
any ranges on the values of the position and orientation variables, except that $z>0$. We start with the following assumptions which enable us to perform symbolic computations and obtain exact analytical expressions:

- Isotropic configurations and corresponding architectures are obtained only for the case when the manipulator is in its home position. The home position is defined as $x=y=0, \alpha=\beta=0$. In other words, displacement along and rotation about only the $\boldsymbol{Z}$ axis is considered.

- The leg lengths have special relationships among themselves. We consider a family of configurations in which alternate legs of the manipulator have equal lengths, i.e., length of the odd numbered legs is $L_{1}$, and that of the even numbered legs $L_{2}=\rho L_{1}$, where $\rho>0$ and in general $\rho \neq 1$. This choice is motivated by the 3 -way symmetry inherent in the manipulator architecture, and the set of configurations is more general than those studied in $[10,11,3,1]$.

These restrictions by no means reflect any limitation of our formulation; relaxing these has only the effect of increasing the complexity of problem ${ }^{5}$.

\subsection{Isotropic configurations}

To ensure the practical utility of the isotropy, we first check for the possible singularities within the target family of configurations. The singularities in statics occur when:

$$
D_{\boldsymbol{H}}=\operatorname{det}\left(\begin{array}{c}
\boldsymbol{H}_{\boldsymbol{F}} \\
\boldsymbol{H}_{\boldsymbol{M}}
\end{array}\right)=0
$$

In this case, the condition becomes:

$$
D_{\boldsymbol{H}}=\frac{54 r_{t}^{3} z^{3} \cos (\gamma-\phi) \sin (\gamma)}{L_{1}^{6} \rho^{3}}, \quad \gamma=\gamma_{b}-\gamma_{t}
$$

\footnotetext{
${ }^{5}$ Although we do not have a proof, we have not been able to find any other family of isotropic configuration (namely with all unequal leg lengths or at $x, y, \alpha, \beta \neq 0$ ) for the SRSPM's studied by us. This is in spite of extensive numerical searches using various methods.
} 
From equation (16), we obtain only two distinct equations defining the leg lengths:

$$
\begin{aligned}
& L_{1}^{2}=1+r_{t}^{2}+z^{2}-2 r_{t} \cos \phi \\
& \rho^{2} L_{1}^{2}=1+r_{t}^{2}+z^{2}-2 r_{t} \cos (2 \gamma-\phi)
\end{aligned}
$$

Eliminating $L_{1}$ between the above equations, we get a linear equation in $\rho^{2}$, which gives the positive solution for $\rho$ as:

$$
\rho=\sqrt{\frac{\left(r_{t}-\cos (2 \gamma-\phi)\right)^{2}+z^{2}+\sin ^{2}(2 \gamma-\phi)}{\left(r_{t}-\cos \phi\right)^{2}+\sin ^{2} \phi+z^{2}}}
$$

The corresponding solution for $L_{1}$ is obtained as:

$$
L_{1}=\sqrt{\left(r_{t}-\cos (2 \gamma-\phi)\right)^{2}+z^{2}+\sin ^{2}(2 \gamma-\phi)}
$$

The expressions for $\rho, L_{1}$ indicate that there are five free parameters, namely $r_{t}, \gamma, \gamma_{t}, \phi$ and $z$, for which the kinematic constraints are valid. We now search for isotropic configurations within this 5-parameter family of kinematically valid configurations. First, we establish the conditions for isotropy in general.

\subsubsection{F-isotropy.}

The kinematically consistent $\boldsymbol{F}$-isotropy conditions computed from equation (12) are found to share a common factor, which can be written as a polynomial in $z_{\boldsymbol{F}}$ :

$$
\begin{aligned}
c_{0} z_{\boldsymbol{F}}^{4} & +c_{1} z_{\boldsymbol{F}}^{2}+c_{2}=0, \text { where } \\
c_{0}= & 2 \\
c_{1}= & 2 r_{t}^{2}-4 \cos (\gamma) \cos (\gamma-\phi) r_{t}+\cos (4 \gamma-2 \phi)+\cos (2 \phi) \\
c_{2}= & (\cos (4 \gamma-2 \phi)+\cos (2 \phi)-2) r_{t}^{2}+4\left(\cos (\phi) \sin ^{2}(2 \gamma-\phi)\right. \\
& \left.+\cos (2 \gamma-\phi) \sin ^{2}(\phi)\right) r_{t}+\cos (4 \gamma-2 \phi)+\cos (2 \phi)-2
\end{aligned}
$$

where $z_{\boldsymbol{F}}$ denotes the height of the centre of the top platform when the manipulator achieves $\boldsymbol{F}$-isotropy. 


\subsection{2 $M$-isotropy.}

In this case also, the isotropy conditions in equation (13) have a common factor, which is a quadratic in $z_{M}^{2}$ :

$d_{0} z_{M}^{4}+d_{1} z_{M}^{2}+d_{2}=0$, where

$d_{0}=2$

$d_{1}=r_{t}^{2}-2 \cos (\gamma) \cos (\gamma-\phi) r_{t}+1$

$d_{2}=-r_{t}^{4}+4 \cos (\gamma) \cos (\gamma-\phi) r_{t}^{3}-2(\cos (2 \gamma)+\cos (2(\gamma-\phi))+1) r_{t}^{2}+4 \cos (\gamma) \cos (\gamma-\phi) r_{t}-1$

where $z_{M}$ denotes the height of the centre of the top platform when the manipulator achieves $M$-isotropy.

\subsubsection{Combined static isotropy.}

The condition for combined static isotropy is simply the intersection of the above two conditions, i.e., $z_{\boldsymbol{F}}=z_{\boldsymbol{M}}$. In other words, equations $(22,23)$ should have common $\operatorname{root}(\mathrm{s})$ in $z^{2}$. The condition for the same can be obtained in closed form by eliminating $z^{2}$ from these equations:

$$
c_{2}^{2} d_{0}^{2}+c_{1} c_{2} d_{0} d_{1}+c_{1}^{2} d_{0} d_{2}-c_{1} d_{1} d_{2}+d_{2}^{2}+c_{2}\left(d_{1}^{2}-2 d_{0} d_{2}\right)=0
$$

The eliminant is of degree 6 in $r_{t}$, but it is possible to write it as $r_{t} \sin ^{2}(\gamma) \sin ^{2}(\gamma-\phi) P_{5}\left(r_{t}\right)$, where the quintic $P_{5}\left(r_{t}\right)=0$, as the vanishing of the other factor leads to singularity. The coefficients of the quintic are functions of the parameters $\gamma, \phi$, and can be derived in closed form. However, due to their large size, we do not include them here. When the quintic has a real solution, equations $(22,23)$ share a common root, and the corresponding positive value 
of $z$ can be obtained as:

$$
\begin{aligned}
& z=\sqrt{\frac{N_{z}}{D_{z}}} \\
& N_{z}=-r_{t}^{4}+4 \cos (\gamma) \cos (\gamma-\phi) r_{t}^{3}-2(2 \cos (2(\gamma-\phi)) \\
& \left.\times \cos ^{2}(\gamma)+\cos (2 \gamma)\right) r_{t}^{2}+2 \cos (\gamma)((2 \cos (2 \gamma)-1) \cos (\gamma-\phi) \\
& +\cos (3(\gamma-\phi))) r_{t}+2 \sin ^{2}(\phi)-\cos (4 \gamma-2 \phi) \\
& D_{z}=r_{t}^{2}-2 \cos (\gamma) \cos (\gamma-\phi) r_{t}+\cos (4 \gamma-2 \phi)+\cos (2 \phi)-1
\end{aligned}
$$

\subsection{Examples of combined static isotropy}

We choose the free parameters as:

$$
\gamma_{b}=\frac{\pi}{5}, \gamma_{t}=\frac{\pi}{10}, \phi=\frac{\pi}{18}
$$

The numerical values of $r_{t}$ are obtained as:

$$
r_{t}=(0.3789,0.9828 \pm 0.1866 i, 1.4795,5.5939)
$$

Of these, only $r_{t}=0.3789$ is feasible for the ranges of parameters we have chosen. The resulting value of $z$ from equation (25) is obtained as $z=0.4627$. The corresponding configuration is shown in figure 3 .

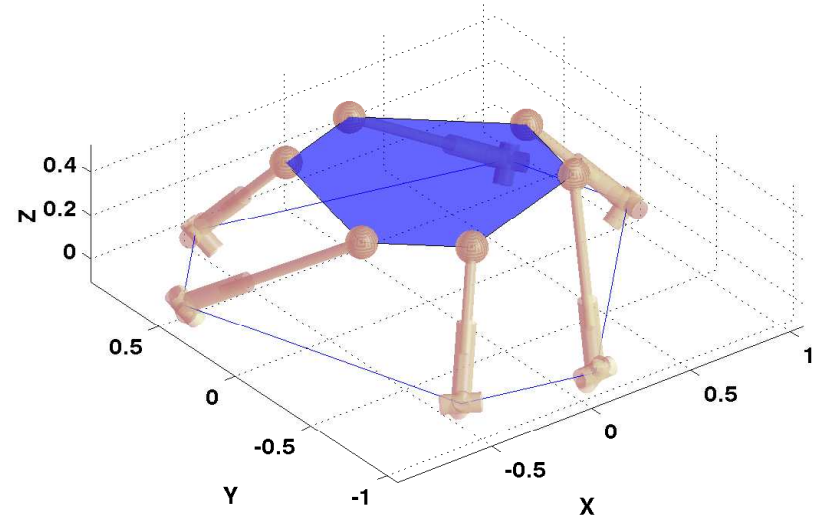

Figure 2: Combined static isotropic configurations of the SRSPM 


\section{Impossibility of spatial isotropy within the family of configurations studied}

In this section, we explore the possibility of identifying a configuration showing spatial isotropy within the three-way symmetric family of configurations studied in this paper. First, we summarise the different kinds of isotropy discussed in literature and their relationships. The matrices $\boldsymbol{J}_{\boldsymbol{\omega}}$ and $\boldsymbol{J}_{\boldsymbol{v}}$ map the active joint rates to the angular velocity $\boldsymbol{\omega}$ and linear

Table 1: Summary of different kinds of isotropy of a six-DOF spatial manipulator

\begin{tabular}{||l|l|l||}
\hline & Type of isotropy & Condition(s) \\
\hline 1 & $\boldsymbol{\omega}$-isotropy & $\boldsymbol{J}_{\boldsymbol{\omega}} \boldsymbol{J}_{\boldsymbol{\omega}}^{T}=\left\|\boldsymbol{\omega}^{*}\right\|^{2} \boldsymbol{I}_{3}$ \\
\hline 2 & $\boldsymbol{v}$-isotropy & $\boldsymbol{J}_{\boldsymbol{v}} \boldsymbol{J}_{\boldsymbol{v}}^{T}=\left\|\boldsymbol{v}^{*}\right\|^{2} \boldsymbol{I}_{3}$ \\
\hline 3 & $\boldsymbol{F}$-isotropy & $\boldsymbol{H}_{\boldsymbol{F}} \boldsymbol{H}_{\boldsymbol{F}}^{T}=\left\|\boldsymbol{F}^{*}\right\|^{2} \boldsymbol{I}_{3}$ \\
\hline 4 & $\boldsymbol{M}$-isotropy & $\boldsymbol{H}_{\boldsymbol{M}} \boldsymbol{H}_{\boldsymbol{M}}^{T}=\left\|\boldsymbol{M}^{*}\right\|^{2} \boldsymbol{I}_{3}$ \\
\hline 5 & Combined kinematic isotropy & Conditions 1 and 2 \\
\hline 6 & Combined static isotropy & Conditions 3 and 4 \\
\hline $7 \mathrm{a}$ & & Condition 5 and $\boldsymbol{J}_{\boldsymbol{\omega}} \boldsymbol{J}_{\boldsymbol{v}}^{T}=\mathbf{0}$, or equivalently, \\
$7 \mathrm{~b}$ & Spatial isotropy & Condition 6 and $\boldsymbol{H}_{\boldsymbol{M}} \boldsymbol{H}_{\boldsymbol{F}}^{T}=\mathbf{0}$, or equivalently, \\
$7 \mathrm{c}$ & & Conditions 5 and 6 \\
\hline
\end{tabular}

velocity $\boldsymbol{J}_{\boldsymbol{v}}$ respectively, and $\left\|\cdot^{*}\right\|$ denotes the constant norm of the corresponding isotropic entity. As seen in table 1, conditions 1 through 4 are independent, i.e., they can be satisfied individually without regard to any of the other conditions. Condition 5 was introduced in [5], while condition 6 is introduced in this paper. As discussed in section 1, condition 7a has been very popular among researches. However, it has been discussed in detail in [5] that conditions 7 have not been achieved in any physically realisable Stewart platform. Further, it was also shown conclusively that it was impossible to find a configuration satisfying condition $7 \mathrm{a}$ among a family of configurations that satisfied condition 5. In the following, we show that it is impossible to satisfy condition $7 \mathrm{~b}$ within the family of configurations described here that 
satisfies condition 6 .

\subsection{Impossibility of satisfying condition $7 \mathrm{~b}$ within the family of configurations considered}

We prove the above following steps similar to those used in [5]. For the sake of brevity, let the length of the odd-, and even-numbered legs be $L$ and $\rho L$ respectively. With these, the expression for $\boldsymbol{H}_{\boldsymbol{M}} \boldsymbol{H}_{\boldsymbol{F}}^{T}$ is obtained as follows:

$$
\boldsymbol{H}_{\boldsymbol{M}} \boldsymbol{H}_{\boldsymbol{F}}^{T}=\left(\begin{array}{ccc}
k_{11} & k_{12} & 0 \\
-k_{12} & k_{11} & 0 \\
0 & 0 & -2 k_{11}
\end{array}\right)
$$

where $k_{11}=\frac{3 z\left(\sin (2 \gamma-\phi)-\rho^{2} \sin \phi\right) r_{t}}{2 L^{2} \rho^{2}}, k_{12}=-\frac{3 z r_{t}\left(-\cos \phi \rho^{2}+r_{t} \rho^{2}-\cos (2 \gamma-\phi)+r_{t}\right)}{2 L^{2} \rho^{2}}$. Condition 7b would be satisfied iff $k_{11}=0=k_{12}$. Setting $k_{11}=0$, we find the positive root for $\rho$ as:

$$
\rho=\sqrt{\frac{\sin (2 \gamma-\phi)}{\sin \phi}}
$$

Equating this with the expression for $\rho$ obtained from inverse kinematics, we get:

$$
2 \sin (\gamma-\phi)\left(\cos \gamma\left(z^{2}+r_{t}^{2}+1\right)-2 \cos (\gamma-\phi) r_{t}\right)=0, \quad \sin \phi \neq 0
$$

Further, substituting the value of $\rho$ from equation (27) in the expression of $k_{12}$ and setting it to zero, we get:

$$
\frac{3 z \sin \gamma r_{t}\left(\cos \gamma-\cos (\gamma-\phi) r_{t}\right)}{L^{2} \sin (2 \gamma-\phi)}=0
$$

We now have two equations, namely $(28,29)$ in the variables $r_{t}$ and $z$. To eliminate $r_{t}$, we first obtain an expression for the positive solution for $r_{t}$ from equation (29):

$$
r_{t}=\frac{\cos \gamma}{\cos (\gamma-\phi)}
$$


Substituting this value in equation (28), we get an equation in $z$ alone:

$$
2\left(\cos \gamma\left(z^{2}+\cos ^{2} \gamma \sec ^{2}(\gamma-\phi)+1\right)-2 \cos \gamma\right) \sin (\gamma-\phi)=0, \quad \sin \phi \sin (2 \gamma-\phi) \neq 0
$$

Solving the last equation for the positive value of $z$, we get:

$$
z=\sqrt{1-\cos ^{2} \gamma \sec ^{2}(\gamma-\phi)}
$$

With these values of $\rho, r_{t}, z$ we calculate the matrix $\boldsymbol{H}_{\boldsymbol{F}} \boldsymbol{H}_{\boldsymbol{F}}^{T}$ as:

$$
\boldsymbol{H}_{\boldsymbol{F}} \boldsymbol{H}_{\boldsymbol{F}}^{T}=\left(\begin{array}{lll}
\frac{3 \cos ^{2} \gamma \sin \gamma \sin \phi}{L^{2} \cos ^{3}(\gamma-\phi)} & 0 & 0 \\
0 & \frac{3 \cos ^{2} \gamma \sin \gamma \sin \phi}{L^{2} \cos ^{3}(\gamma-\phi)} & 0 \\
0 & 0 & \frac{6 \cos ^{2} \gamma \sin \gamma \sin \phi}{L^{2} \cos (\gamma-\phi)}
\end{array}\right)
$$

The isotropy condition in this case reduces to the single equation:

$$
\cos 2(\gamma-\phi)=0
$$

Therefore, condition 3 is satisfied when we have:

$$
\phi=\gamma \pm \frac{\pi}{4}
$$

With the same values of the parameters, the matrix $\boldsymbol{H}_{\boldsymbol{M}} \boldsymbol{H}_{\boldsymbol{M}}^{T}$ is calculated as:

$$
\boldsymbol{H}_{\boldsymbol{M}} \boldsymbol{H}_{\boldsymbol{M}}^{T}=\frac{3 \sin (\gamma) \sin (\phi)}{L^{2} \cos (\gamma-\phi)}\left(\begin{array}{lll}
1 & 0 & 0 \\
0 & 1 & 0 \\
0 & 0 & 2
\end{array}\right)
$$

It is very clear from the above expression that condition 4 cannot be satisfied for the parameter values for which condition 3 is satisfied. Therefore it is impossible to find spatial isotropy within the family of configurations under investigation. Further, given the impossibility of meeting either of conditions $7 \mathrm{a}, 7 \mathrm{~b}$ within this family, it is logically impossible to satisfy condition 7c within the same set. Analytical and numerical attempts (not included here for 
the sake of brevity) to find real, finite solutions to the combined conditions 5,6 confirm this conclusion.

We can summarise the findings of this section as follows: it is impossible to obtain a spatially isotropic SRSPM within the family of configurations considered in this paper ${ }^{6}$.

\section{Design of an SRSPM for combined static isotropy}

The formulation presented in this paper allow us to solve the problems of analysis and synthesis within the same setup, in addition to studying the isotropic configurations in general. In this context, by analysis we mean obtaining the isotropic configurations of a manipulator with a given architecture, and by synthesis, the determination of the architecture parameters such that the manipulator is isotropic at a given configuration. We present a few case studies below.

\subsection{Synthesis of an SRSPM for combined static isotropy at a given position $z_{0}$ and orientation $\phi_{0}$}

In this case we assume that the top platform location and orientation have been completely specified by $z_{\boldsymbol{F}}=z_{\boldsymbol{M}}=z_{0}$ and $\phi=\phi_{0}$ (in conjunction with the assumptions defining the isotropic family). The task is to obtain $\gamma$ and $r_{t}$ such that the manipulator exhibits combined static isotropy.

We start with the $\boldsymbol{F}$-isotropy equation (22) and the $\boldsymbol{M}$-isotropy equation (23). Substituting the actual expressions of $c_{i}, d_{i}$ in these equations, and rewriting them as polynomial equations in $r_{t}$, we get a quartic and a quadratic respectively:

$$
\begin{aligned}
& g_{0} r_{t}^{4}+g_{1} r_{t}^{3}+g_{2} r_{t}^{2}+g_{3} r_{t}+g_{4}=0 \\
& h_{0} r_{t}^{2}+h_{1} r_{t}+h_{2}=0
\end{aligned}
$$

\footnotetext{
${ }^{6}$ In theory, such configurations may exist outside the family studied here. However, extensive numerical searches have failed to obtain any solution to this problem.
} 
The common root of these two equations can be obtained in terms of the coefficients $g_{i}, h_{i}$ when the resultant with respect to $r_{t}$ vanishes. The resultant is a complicated expression involving trigonometric terms in $\gamma$, and algebraic terms in $z_{0}$. We transform it to a polynomial in $t=\tan (\gamma / 2)$ and simplify its coefficients using the algorithms described in [9]. This results in a $32^{\text {nd }}$ degree polynomial in $t$. Extracting the real values of $t$ such that the corresponding values of $\gamma$ are within the prescribed limits, we compute $r_{t}$ numerically. For every positive solution for $r_{t}$ within the specified range, the free parameter $\gamma_{t}$ can be chosen as convenient, and the architecture of the manipulator can be completely prescribed. We illustrate this synthesis procedure with an example below.

We choose the configuration as $z_{0}=1 / 2, \phi_{0}=\pi / 20$, and the free architecture parameter as $\gamma_{t}=\pi / 12$. Corresponding to these numerical values, there are 24 real solutions for $t$, of which, however, only 2 turn out to be feasible. The feasible values of $\gamma$ are $(-0.1750,0.3321)$ and the corresponding values of $r_{t}$ are $(0.3239,0.3239)$ respectively. The configurations are shown in figures $3(\mathrm{a})-3(\mathrm{~b})$.

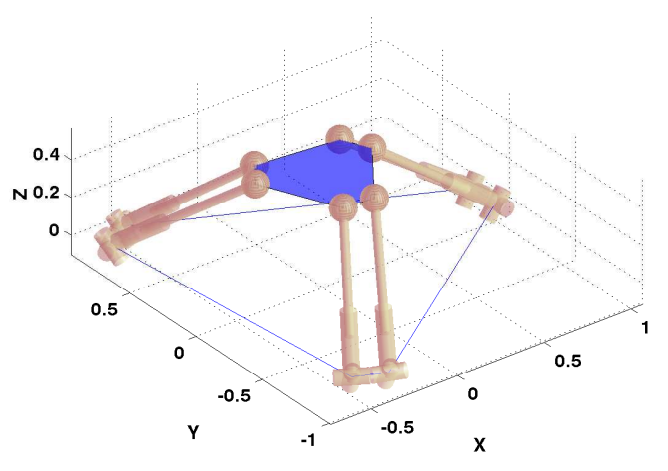

(a) $\gamma=-0.1750, r_{t}=0.3239$

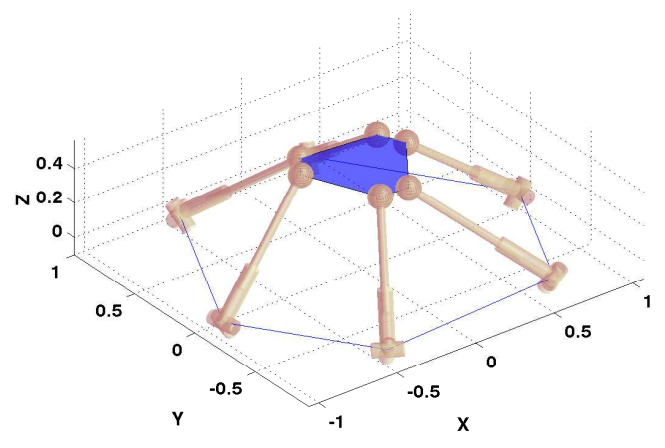

(b) $\gamma=0.3321, r_{t}=0.3239$

Figure 3: Combined static isotropy of an SRSPM at a given location and orientation 


\subsection{Combined static isotropic configurations of an SRSPM of given architecture}

In this section, we find out the configurations of an SRSPM of given architecture showing combined static isotropy. The manipulator geometry is completely specified in terms of the architecture variables, $r_{t}, \gamma$ and $\gamma_{t}$. We need to find the configuration variables $z$ and $\phi$ such that the conditions for combined static isotropy are met.

We refer to the condition for combined static isotropy in equation (24), which is a function of $\phi$ alone. We convert this equation into a polynomial in $u=\tan (\phi / 2)$ using the symbolic simplification tools as in the case of the synthesis. In this case we end up with a $8^{\text {th }}$ degree polynomial in $u$. For each of the feasible values of $\phi$ arising from the solutions for $u$, the corresponding value of $z$ can be computed uniquely from equation (25), thereby completing the specification of the manipulator configuration. We demonstrate the solution procedure with an example below.

We use an architecture based on the INRIA prototype of the SRSPM (data adopted from [12]). However, we take the top platform to be the moving one. In our notation, the architecture parameters are calculated as:

$$
r_{t}=0.5803, \gamma_{b}=0.2985, \gamma_{t}=0.6573
$$

The isotropic configurations are shown in figures 4(a)-4(b).

\section{Conclusion}

In this paper, we have developed an algebraic formulation for the study of static isotropy of spatial manipulators. We have applied the theory to SRSPM's, and obtained in closed-form a family of configurations showing force and moment isotropy simultaneously. The formulation allows us to design an SRSPM for combined static isotropy at a given configuration within this family. It also allows us to obtain such configurations for an existing SRSPM with a given architecture. The analytical procedures and results presented in the paper have been 


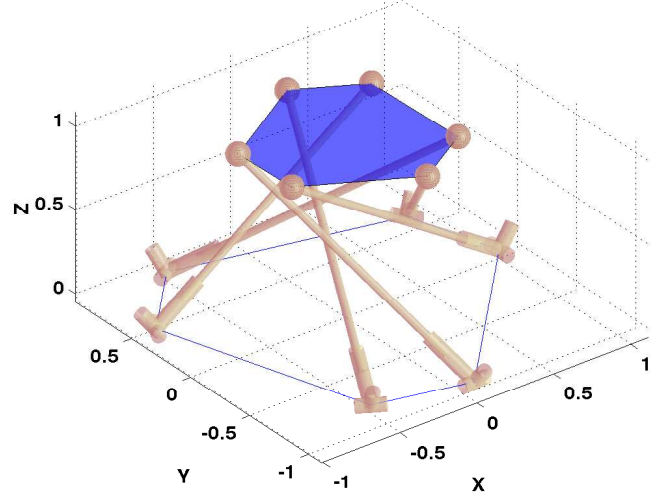

(a) $z=1.0216, \phi=-2.7254$

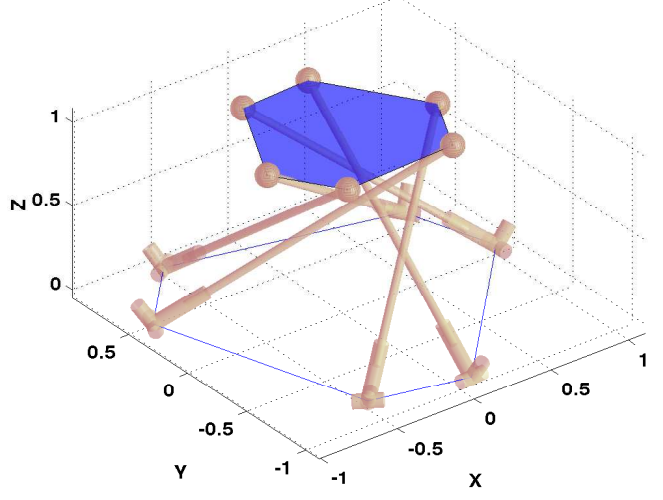

(b) $z=1.0216, \phi=2.0078$

Figure 4: Combined static isotropic configurations of the SRSPM with INRIA geometry

numerically illustrated with examples of both analysis and design. However, it is found that it is impossible to attain spatial isotropy within the family of SRSPM showing combined static isotropy.

\section{Acknowledgment}

The authors wish to thank the anonymous reviewers for their comments, which have helped improve the paper.

\section{References}

[1] K. Y. Tsai and K. D. Huang, "The design of isotropic 6-DOF parallel manipulators using isotropy generators," Mechanism and Machine Theory, vol. 38, pp. 1199-1214, 2003.

[2] K. E. Zanganeh and J. Angeles, "Kinematic isotropy and the optimum design of parallel manipulators," International Journal of Robotics Research, vol. 16, pp. 185-197, April 1997. 
[3] A. Fattah and A. M. H. Ghasemi, "Isotropic design of spatial parallel manipulators," International Journal of Robotics Research, vol. 21, pp. 811-824, September 2002.

[4] Y. X. Su, B. Y. Duan, and C. H. Zheng, "Genetic design of kinematically optimal fine tuning Stewart platform for large spherical radio telescope," Mechatronics, vol. 11, pp. 821-835, 2001.

[5] S. Bandyopadhyay and A. Ghosal, "An algebraic formulation of kinematic isotropy and design of isotropic 6-6 Stewart platform manipulators," Mechanism and Machine Theory, vol. 43, pp. 591-616, May 2008.

[6] R. A. Horn and C. A. Johnson, Matrix Analysis. Cambridge University Press, 1985.

[7] I. N. Herstein, Topics in Algebra. New York: John Wiley \& Sons, 1975.

[8] O. Ma and J. Angeles, "Architectural singularities of platform manipulators," in Proceedings of the 1991 IEEE International Conference on Robotics and Automation, pp. 1542-1547, 1991.

[9] S. Bandyopadhyay and A. Ghosal, "Geometric characterization and parametric representation of the singularity manifold of a 6-6 Stewart platform manipulator," Mechanism and Machine Theory, vol. 41, pp. 1377-1400, Nov. 2006.

[10] C. A. Klein and T. A. Miklos, "Spatial robotic isotropy," International Journal of Robotics Research, vol. 10, pp. 426-437, August 1991.

[11] J. Angeles, "The design of isotropic manipulator architectures in the presence of redundancies," International Journal of Robotics Research, vol. 11, pp. 196-202, June 1992.

[12] H. Li, C. Gosselin, M. J. Richard, and B. M. St-Onge, "Analytic form of the sixdimensional singularity locus of the general Gough-Stewart platform," in Proceedings of ASME 28th Biennial Mechanisms and Robotics Conference, Salt Lake City, Utah, USA, September 2004. 


\section{A Real linear maps and geometry of the range space}

Let us consider the real linear map $f: \mathbb{R}^{m} \rightarrow \mathbb{R}^{n}$ which takes $\boldsymbol{x}$ to $\boldsymbol{A} \boldsymbol{x}$. We wish to obtain the distribution of all possible $\boldsymbol{y}=\boldsymbol{A} \boldsymbol{x}$ for any $\boldsymbol{x} \in \mathbb{R}^{m}$. This can be done by studying the possible values of norm of $\boldsymbol{y}$, which can be computed from the following:

$$
\|\boldsymbol{y}\|^{2}=\boldsymbol{x}^{T} \boldsymbol{A}^{T} \boldsymbol{A} \boldsymbol{x}=\boldsymbol{x}^{T} \boldsymbol{Q} \boldsymbol{x}
$$

where $\boldsymbol{Q}=\boldsymbol{Q}^{T} \in \mathbb{R}^{m \times m}$. Without any loss of generality, we study the maps of the unit sphere $\mathbb{S}^{n-1}$ alone ${ }^{7}$, i.e., we restrict ourselves to $\|\boldsymbol{x}\|=1$. The extreme values of $\|\boldsymbol{y}\|^{2}$ can therefore be obtained by obtaining the extrema of the following function:

$$
h(\boldsymbol{x})=\boldsymbol{x}^{T} \boldsymbol{Q} \boldsymbol{x}+\lambda\left(1-\boldsymbol{x}^{T} \boldsymbol{x}\right)
$$

where $\lambda$ represents Lagrange's undetermined multiplier. After differentiation with respect to $\boldsymbol{x}$ and rearrangement, we obtain the following eigenproblem:

$$
\boldsymbol{Q x}=\lambda \boldsymbol{x}
$$

It is well known in linear algebra(see, e.g.,[6]), that $\lambda \in \mathbb{R}$ and $\lambda \geq 0$ as $\boldsymbol{Q}$ is symmetric, and $\boldsymbol{x} \in \mathbb{R}^{m}$. If $\left(\lambda_{i}^{*}, \boldsymbol{x}_{i}^{*}\right)$ form an eigen-pair satisfying equation (40), extreme values of $\|\boldsymbol{y}\|$ occur when:

$$
\boldsymbol{y}_{i}^{*}=\boldsymbol{A} \boldsymbol{x}_{i}^{*} \quad i=1, \ldots, n
$$

and the extreme values are:

$$
\left\|\boldsymbol{y}_{i}^{*}\right\|=\sqrt{\boldsymbol{x}_{i}^{* T} \boldsymbol{Q} \boldsymbol{x}_{i}^{*}}=\sqrt{\lambda_{i}^{*}} \quad i=1, \ldots, n
$$

Finally, one can write:

$$
\boldsymbol{y}_{i}^{*}=\sqrt{\lambda_{i}^{*}} \boldsymbol{x}_{i}^{*}
$$

\footnotetext{
${ }^{7}$ The results obtained thus can be appropriately scaled when $\|\boldsymbol{x}\| \neq 1$.
} 
Equation (42) allows a direct study of the conditioning of the matrix $\boldsymbol{A}$ (and therefore the nature of the map $f$ ), including defining the exact criteria for the two extreme cases: singularity and isotropy. If all $\lambda_{i} \neq 0$ then it is well known that $\boldsymbol{y}$ lies on a $n$-dimensional (hyper)-ellipsoid, whose axes are defined by $\boldsymbol{y}_{i}^{*}$, i.e., axes lengths are given by $2 \sqrt{\lambda_{i}^{*}}$ and corresponding axes are aligned with $\boldsymbol{y}_{i}^{*}$. If $m<n$, then $(n-m)$ eigenvalues are always zero, and the dimension of the ellipsoid is restricted to $m$. However, if $p$ additional eigenvalues are zeros, the ellipsoid loses $p$ dimensions, and reduces to an ellipse, a line through the origin, and the origin respectively when $(\min (m, n)-p)=2,1,0$. The matrix is termed singular when one or more of $\lambda_{i}$ 's vanish.

On the other extreme, if $\lambda_{i}=\lambda_{j}(=\bar{\lambda}$ say $), i \neq j$, then the the ellipsoid cuts the planes parallel to $\boldsymbol{y}_{i}, \boldsymbol{y}_{j}$ in circles of radius $\bar{\lambda}$. Similarly, if all the eigenvalues are equal $\bar{\lambda}$, then the ellipsoid reduces to the sphere $\mathbb{S}^{n-1}$ scaled by $\sqrt{\bar{\lambda}}$. Under this condition, the matrix $\boldsymbol{Q}$ equals $\bar{\lambda} \boldsymbol{I}_{n}$, where $\boldsymbol{I}_{n}$ denotes the $n \times n$ identity matrix. The singular values of $\boldsymbol{A}$ equals $\lambda_{i}$ in general, and in this case, the condition number of $\boldsymbol{A}$, defined as the ratio of the largest singular value to the smallest one, is seen to reduce to unity. The matrix is termed isotropic in this case.

\section{B Symbolic construction of the characteristic polyno- mial of a square matrix}

The characteristic polynomial of a square matrix $\boldsymbol{A}$ of dimension $n$ is defined as

$$
\begin{aligned}
P_{n}(\lambda) & =\operatorname{det}\left(\lambda \boldsymbol{I}_{n}-\boldsymbol{A}\right) \\
& =\lambda^{n}+a_{1} \lambda^{n-1}+\cdots+a_{n-1} \lambda+a_{n}
\end{aligned}
$$

However, construction of the polynomial from this definition requires symbolic expansion of the determinant, which is computationally very expensive, and can indeed be prohibitive. Fortunately, we can compute the coefficients $a_{i}$ above directly using a simple formula derived below from the Newton's identity. 
Let $s_{k}=\sum_{i=1}^{n} \lambda_{i}^{k}, k=1, \ldots, n$, where $\lambda_{i}$ is a root of equation (43). Then for $k=1, \ldots, n$, Newton's identity states that, for $k=1, \ldots, n$,

$$
s_{k}+a_{1} s_{k-1}+a_{2} s_{k-2}+\cdots+a_{n-1} s_{1}+k a_{k}=0
$$

Noting that if $\boldsymbol{A} \boldsymbol{x}=\lambda \boldsymbol{x}$, then for any positive integer $i$, we have $\boldsymbol{A}^{i} \boldsymbol{x}=\lambda^{i} \boldsymbol{x}$, and that for any square matrix the trace equals the sum of its eigenvalues, we get the relation $s_{k}=\operatorname{tr}\left(\boldsymbol{A}^{k}\right)$, $k=1, \ldots, n$. Finally, substituting $s_{k}$ in equation (44) we get an explicit formula for $a_{k}$ as follows:

$$
a_{k}= \begin{cases}-\operatorname{tr}(\boldsymbol{A}) & k=1 \\ \frac{(-1)}{k}\left(\operatorname{tr}\left(\boldsymbol{A}^{k}\right)+\sum_{i=1}^{k-1} a_{i} \operatorname{tr}\left(\boldsymbol{A}^{k-i}\right)\right) & k=2, \ldots, n\end{cases}
$$

The above method involves mostly the computation of the traces of $\boldsymbol{A}^{k}$, and it is therefore much more economical than the explicit expansion of the determinant. The complexity of the algorithm can be further reduced by taking advantage of any special structure of $\boldsymbol{A}$, such as bandedness, symmetry or skew-symmetry. 